\title{
Comparative anticancer activity of dolaborane diterpenes from the roots of Ceriops tagal (Rhizophoraceae)
}

\author{
Musa CHACHA \\ Institute of Marine Sciences, University of Dar es Salaam, P.O. Box 668, Zanzibar, Tanzania. \\ E-mail: chacha@ims.udsm.ac.tz
}

\begin{abstract}
This study aimed at investigating the anticancer activity of tagalsins A, B, C, D, E, F and G isolated from the roots of Ceriops tagal. Their structures were established based on the IR, MS and NMR spectral data. Anticancer activity was evaluated using caspase-3 colourimetric assays and the minimum activation concentrations were established. The study revealed that tagalsin A, B, C, D, E, F and G induces apoptosis through the activation of caspase-3 enzyme. Tagalsin A was the most active (MAC value of $0.12 \mu \mathrm{g} / \mathrm{mL}$ ) followed by tagalsin B (MAC of $0.15 \mu \mathrm{g} / \mathrm{mL}$ ) and and tagalsin E was the least active (MAC value of 1.16 $\mu \mathrm{g} / \mathrm{mL}$ ). Structure activity relationship revealed that epoxide rings and oxygenation enhanced the activity of tagalsins in inducing caspase- 3 activation of apoptotic pathway, thus, providing an avenue for the development of anticancer agents with novel mechanism of actions.
\end{abstract}

(C) 2012 International Formulae Group. All rights reserved.

Keywords: Ceriops tagal, dolaboranes, tagalsins, caspase-3.

\section{INTRODUCTION}

One of the hallmarks of cancer is its resistance to apoptosis which is important in the development and maintenance of higher organisms (Hanahan and Weinberg, 2000). This resistance enables cancerous cells to survive and divide even in the presence of endogenous proapoptotic stimuli (Peterson et al., 2009). In human cells, apoptosis involves the activation of a unique family of proteases, the caspases (cysteine-containing aspartatespecific proteases) which plays a central role in initiation and execution of the apoptotic process. The caspases are constitutively expressed in all cell types as zymogens, or procaspases, that has to be proteolytically cleaved to become active enzymes. Unlike normal cells, cancerous cells evades apoptosis through variety of mechanisms involving mutation or altered expression levels of key proteins in the apoptotic cascade (Igney and Krammer, 2002; Lowe et al., 2004) including mutation of p53 (Vogelstein and Kinzler, 2001), elevated expression of the antiapoptotic proteins in the Bcl-2 family (Kirkin et al., 2004) and inactivation of Apaf-1 (Soengas et al., 2001). The net result of these alterations is a resistance to apoptosis, allowing for unchecked cellular proliferation.

The direct induction of apoptosis has therefore emerged as a powerful anticancer strategy, and compounds that activate caspase enzymes in the apoptotic pathway have great 
potential as novel chemotherapeutic agents. It is in this vein that tagalsin A (1), B (2), C (3), $\mathrm{D}(4), \mathrm{E}(5), \mathrm{F}(6)$ and $\mathrm{G}(7)$ isolated from the roots of Ceriops tagal were evaluated for anticancer activity using caspase-3 calorimetric assay.

\section{MATERIALS AND METHODS General}

NMR spectra were determined on a Bruker Avance DRX 600 and referenced to residual solvent signals. Low-resolution mass spectra were obtained on Finnigan MAT $\mathrm{LCQ}^{\mathrm{DECA}}$ instrument and high resolution mass spectra were obtained on GCT Premier Instrument. The ultraviolet and visible (UVVIS) spectra were taken on Shimadzu UV2101PC UV-Vis Scanning Spectrophotometer. Infrared (IR) absorptions were measured on a Perkins Elmer System $2000 \quad$ FT-IR Spectrophotometer using $\mathrm{KBr}$ pellets. Specific rotations $[\alpha]_{D}^{25}$ were measured on polatronicD $($ Schmidt + Haensch $)$ polarimeter. Melting points were recorded using Stuart Scientific melting point apparatus and are uncorrected.

\section{Plant material}

The roots of Ceriops tagal were collected from Kisakasaka Mangrove Reserve, Zanzibar, Tanzania. The plant species was identified by Mtumwa Mhidini of the Institute of Marine Sciences, University of Dar es Salaam. A voucher specimen (CTM-104) was deposited at the laboratory of Natural Products and Biotechnology of the Institute of Marine Sciences, University of Dar es Salaam.

\section{Extraction, isolation and identification of compounds}

The roots of Ceriops tagal were air dried and then pulverized. The powdered sample $(3.8 \mathrm{Kg})$ was extracted using $\mathrm{CHCl}_{3}$ thrice at room temperature and the extract concentrated in vacuum to give a brown residue. The extract (22 g) was adsorbed in silica gel, loaded on a silica gel chromatographic column and eluted with $n$ hexane- $\mathrm{CHCl}_{3}$ as a gradient to obtain twelve fractions which were combined based on the TLC analysis. Fraction B (1.34 g) was adsorbed in silica gel and applied to a silica gel column eluted with $n$-hexane/ $\mathrm{Et}_{2} \mathrm{O}$ (13:1.5) to obtain tagalsin F (6) (132 mg). Fraction D $(0.8 \mathrm{~g})$ was subjected to a Sephadex ${ }^{\circledR}$ LH-20 column and eluted with $\mathrm{CHCl}_{3}-\mathrm{MeOH}(1: 1)$ to give tagalsins $\mathrm{E}(\mathbf{5})(17 \mathrm{mg}), \mathrm{C}$ (3) (18.6 $\mathrm{mg}$ ) and $\mathrm{G}(7)(14.2 \mathrm{mg})$. Fraction $\mathrm{E}(60 \mathrm{mg}$, 10:1) was adsorbed in silica gel and loaded on the silica gel column and eluted with $n$ hexane/ $\mathrm{Et}_{2} \mathrm{O}(8: 1)$ to afford tagalsins $\mathrm{A}(\mathbf{1})$ (16.6 mg), B (2) (19.5 mg), and D (4) (14.2 $\mathrm{mg}$ ). Tagalsin A (1) was recrystallized as needles from acetone.

Spectral data of dolaboranes 1, 2, 3, 4, 5, 6 and 7

Tagalsin A (1)

Pale yellow needle crystals. m.p. 68-71 ${ }^{\circ} \mathrm{C} ;[\alpha]_{D}^{2 \mathrm{~s}}+69.24^{\circ}\left(c 0.052, \mathrm{CHCl}_{3}\right) ; \mathrm{IR}(\mathrm{KBr})$ $v_{\max } 3409,2859,1672,1412 \mathrm{~cm}^{-1}$; EI-MS $\mathrm{m} / \mathrm{z}$ $(\%): 316[\mathrm{M}]^{+}(15), 286$ (17), 259 (20), 179 (27), $163 \quad$ (53), $136 \quad$ (100), $107 \quad$ (93); HRFABMS $\mathrm{m} / \mathrm{z} 317.21110[\mathrm{M}+\mathrm{H}]^{+}$(calcd for $\left.\mathrm{C}_{20} \mathrm{H}_{29} \mathrm{O}_{3}, 317.21111\right)$. ${ }^{1} \mathrm{H}-\mathrm{NMR} \delta(\mathrm{ppm})$ : $6.35(1 \mathrm{H}, d, J=6.8 \mathrm{~Hz}, \mathrm{H}-1), 5.80(1 \mathrm{H}, d d, J$ $=10.5,17.5 \mathrm{~Hz}, \mathrm{H}-15), 4.93(1 \mathrm{H}, d, J=17.5$ $\mathrm{Hz}, \mathrm{H}-16), 4.85$ (1H, $d, J=10.5 \mathrm{~Hz}, \mathrm{H}-16)$, $3.43(1 \mathrm{H}, d, J=6.0 \mathrm{~Hz}, \mathrm{H}-18), 2.95(1 \mathrm{H}, d, J$ $=6.0 \mathrm{~Hz}, \mathrm{H}-18), 2.15(1 \mathrm{H}, d, J=6.8 \mathrm{~Hz}, \mathrm{H}-$ 10), $1.52(1 \mathrm{H}, d d d, J=3.0,4.0,12.5 \mathrm{~Hz}, \mathrm{H}-$ 11), 1.48 (2H, $m, \mathrm{H}-7,12), 1.45$ (1H, $m, \mathrm{H}-6)$, $1.41(1 \mathrm{H}, d d, J=10.5,12.5 \mathrm{~Hz}, \mathrm{H}-11), 1.34$ $(1 \mathrm{H}, d d, J=11.5,12.5 \mathrm{~Hz}, \mathrm{H}-14), 1.21(1 \mathrm{H}$, $d d, J=3.0,14.0 \mathrm{~Hz}, \mathrm{H}-12), 1.18(1 \mathrm{H}, m, \mathrm{H}-6)$, 1.17 (1H, $s, \mathrm{H}-19), 1.06$ (1H, $m, \mathrm{H}-7), 1.05$ (1H, $m, \mathrm{H}-17), 1.02$ (1H, $m, \mathrm{H}-14), 0.79(1 \mathrm{H}$, $s, \mathrm{H}-20) .{ }^{13} \mathrm{C}-\mathrm{NMR} \delta$ (ppm): 190.8 (C-3), 150.3 (C-15), 146.9 (C-2), 120.8 (C-1), 108.5 (C-16), 60.3 (C-4), 54.5 (C-10), 50.4 (C-18), 40.6 (C-8), 39.8 (C-9), 39.5 (C-14), 36.4 (C13), 35.9 (C-5), 34.9 (C-11), 33.9 (C-6), 31.8 (C-12), 31.6 (C-19), 27.3 (C-7), 22.8 (C-17), $12.1(\mathrm{C}-20)$. 


\section{Tagalsin B (2)}

White solid. m.p. $66-69{ }^{\circ} \mathrm{C} ; \quad[\alpha]_{\mathrm{D}}^{25}$ $+164^{\circ}\left(c 0.05, \mathrm{CHCl}_{3}\right)$; IR $(\mathrm{KBr}) v_{\max } 3430$, 2922, 2853, 1681, 1636, 1599, 1219. EIMS $\mathrm{m} / \mathrm{z}(\%): 316[\mathrm{M}]^{+}(17), 283$ (20), 255 (18), 175 (49), 136 (63), 107 (100), 81 (69), 67 (43), 55 (50); HRFABMS $m / z$ 317.2110 [M + $\mathrm{H}]^{+}$(calcd for $\left.\mathrm{C}_{20} \mathrm{H}_{29} \mathrm{O}_{3} 317.2111\right) .{ }^{1} \mathrm{H}-\mathrm{NMR} \delta$ (ppm): $6.31(1 \mathrm{H}, d, J=6.5 \mathrm{~Hz}, \mathrm{H}-1), 5.87$ $(1 \mathrm{H}, d d, J=10.5,17.5 \mathrm{~Hz}, \mathrm{H}-15), 4.92(1 \mathrm{H}, d$, $J=17.5 \mathrm{~Hz}, \mathrm{H}-16), 4.85(1 \mathrm{H}, d, J=10.5 \mathrm{~Hz}$, $\mathrm{H}-16), 3.13(1 \mathrm{H}, d, J=6.0 \mathrm{~Hz}, \mathrm{H}-18), 3.09$ $(1 \mathrm{H}, d, J=6.0 \mathrm{~Hz}, \mathrm{H}-18), 2.19(1 \mathrm{H}, d, J=6.5$ $\mathrm{Hz}, \mathrm{H}-10), 1.60$ (1H, $m, \mathrm{H}-6), 1.56(1 \mathrm{H}, m, \mathrm{H}-$ 11), 1.46 (2H, $m, \mathrm{H}-8,12), 1.43(1 \mathrm{H}, m, \mathrm{H}-$ 11), $1.34(1 \mathrm{H}, d d, J=13.0,13.5 \mathrm{~Hz}, \mathrm{H}-14)$, 1.25 (1H, $m, \mathrm{H}-12), 1.23(1 \mathrm{H}, m, \mathrm{H}-7), 1.21$ (1H, $s, \mathrm{H}-19), 1.18$ (2H, $m, \mathrm{H}-6,7), 1.05(1 \mathrm{H}$, $s, \mathrm{H}-17), 0.72(1 \mathrm{H}, s, \mathrm{H}-20) .{ }^{13} \mathrm{C}-\mathrm{NMR} \delta$ (ppm): 191.8 (C-3), 150.3 (C-15), 147.3 (C-2), 120.2 (C-1), 108.6 (C-16), 61.3 (C-4), 54.8 (C-10), 55.4 (C-18), 40.1 (C-8), 39.3 (C-14), 39.0 (C-9), 36.9 (C-5), 35.9 (C-13), 34.7 (C11), 31.9 (C-6), 31.7 (C-12), 29.5 (C-19), 27.0 (C-7), 22.7 (C-17), 13.1 (C-20).

\section{Tagalsin C (3)}

Yellow oil. $[\alpha]_{D}^{25}+92.3^{\circ} \quad(c 0.05$; $\mathrm{CHCl}_{3}$ ); IR (KBr) $v_{\max } 3403,3082,2924$, 2859, 1662, 1605, 1459, 1411, 1220, $1082 \mathrm{~cm}^{-}$ 1. EI-MS m/z (\%): $300[\mathrm{M}]^{+}(26), 163(35)$, 151 (45), 138 (77), 107 (100), 93 (60), 79 (77); HRFABMS $m / z 301.2162[\mathrm{M}+\mathrm{H}]^{+}$ (calcd for $\mathrm{C}_{20} \mathrm{H}_{29} \mathrm{O}_{2}, 301.2162$ ). ${ }^{1} \mathrm{H}-\mathrm{NMR} \delta$ (ppm): 6.26 (1H, brs, H-18b), $6.22(1 \mathrm{H}, d, J=$ $6.7 \mathrm{~Hz}, \mathrm{H}-1), 5.80(1 \mathrm{H}, d d, J=17.5,10.8 \mathrm{~Hz}$, H-15), 5.43 (1H, brs, H-18a), 4.90 (1H, $d, J=$ $17.5 \mathrm{~Hz}, \mathrm{H}-16 \mathrm{~b}), 4.87(1 \mathrm{H}, d, J=10.8 \mathrm{~Hz}, \mathrm{H}-$ 16a), $2.20(1 \mathrm{H}, d d d, J=14.5,2.5,2.5 \mathrm{~Hz}, \mathrm{H}-$ 6b), $2.08(1 \mathrm{H}, d, J=6.7 \mathrm{~Hz}, \mathrm{H}-10), 1.48(1 \mathrm{H}$, $m$, H-6a \& H-12b), 1.53 (1H, $m$, H-11b), 1.46 (1H, $m, \mathrm{H}-8), 1.34$ (1H, m, H-11a), $1.32(1 \mathrm{H}$, $m, \mathrm{H}-7 \mathrm{~b}), 1.20$ (1H, $m, \mathrm{H}-7 \mathrm{a} \& \mathrm{H}-12 \mathrm{a}), 1.30$ (1H, $m, \mathrm{H}-14 \mathrm{~b}), 1.10$ (1H, $m, \mathrm{H}-14 \mathrm{a}), 1.14$ (3H, s, H-19), 1.05 (3H, s, H-13), 0.64 (3H, $s$, $\mathrm{H}-20) .{ }^{13} \mathrm{C}-\mathrm{NMR} \delta$ (ppm): 185.3 (C-3), 150.8 (C-15), 148.8 (C-4), 147.2 (C-2), 118.9 (C-
18), 118.2 (C-1), 108.9 (C-16), 55.5 (C-10), 41.2 (C-5), 40.8 (C-8 \& C-9), 39.4 (C-14), 36.6 (C-6), 36.5 (C-13), 35.2 (C-11),33.9 (C19), 31.6 (C-12), 25.4 (C-7), 22.9 (C-17), 12.0 (C-20).

\section{Tagalsin D (4)}

White solid. m.p. $64-66{ }^{\mathrm{o}} \mathrm{C} ;[\alpha]_{D}^{25}$ $+38.4^{\circ}\left(c 0.074 ; \mathrm{CHCl}_{3}\right)$; IR ( $\left.\mathrm{KBr}\right) v_{\max } 3406$, 3059, 2924, 2860, 1635, 1476, 1450, 1373, 1264, $943 \mathrm{~cm}^{-}{ }^{\text {; }}$ EI-MS m/z (\%): $304\left[\mathrm{M}^{+}{ }^{+}\right.$(5), 289 (12), 274 (33), 259 (26), 241 (34), 189 (25), 147 (32), 133 (46), 121 (51),107 (98); HRFABMS $m / z 305.2474[\mathrm{M}+\mathrm{H}]^{+}$(calcd for $\left.\mathrm{C}_{20} \mathrm{H}_{33} \mathrm{O}_{2}, 305.2475\right) .{ }^{1} \mathrm{H}-\mathrm{NMR} \delta$ (ppm): 5.80 $(1 \mathrm{H}, d d, J=17.5,10.8 \mathrm{~Hz}, \mathrm{H}-15), 4.90(1 \mathrm{H}, d$, $J=17.5 \mathrm{~Hz}, \mathrm{H}-16 \mathrm{~b}), 4.84(1 \mathrm{H}, d, J=10.8 \mathrm{~Hz}$, $\mathrm{H}-16 \mathrm{a}), 3.45(1 \mathrm{H}, d d, J=2.0,1.5, \mathrm{H}-3), 3.08$ $(1 \mathrm{H}, d, J=4.6 \mathrm{~Hz}, \mathrm{H}-18 \mathrm{~b}), 2.72(1 \mathrm{H}, d, J=$ 4.6, H-18a), 2.15 (1H, m, H-2b), $2.04(1 \mathrm{H}$, $d d d, J=14.0,4.5,4.0 \mathrm{~Hz}, \mathrm{H}-1 \mathrm{~b}), 1.82(1 \mathrm{H}, m$, $\mathrm{H}-1 \mathrm{a}), 1.80(1 \mathrm{H}, m, \mathrm{H}-2 \mathrm{a}), 1.78(1 \mathrm{H}, m, \mathrm{H}-$ 11b), $1.59(1 \mathrm{H}, d d d, J=14.5,3.0,2.5 \mathrm{~Hz}, \mathrm{H}-$ 6b), 1.47 (1H, ddd, $J=13.5,13.5,4.0, \mathrm{H}-12 \mathrm{~b})$, 1.39 (1H, m, H-10), 1.39 (3H, $s, \mathrm{H}-19) .1 .33$ $(1 \mathrm{H}, m, \mathrm{H}-8), 1.32(1 \mathrm{H}, d d, J=13.0,12.0 \mathrm{~Hz}$, 14b), 1.25 (1H, $m, \mathrm{H}-12 \mathrm{a}), 1.18(1 \mathrm{H}, d d d, J=$ 13.5, 13.5, 4.0 Hz, H-11a), 1.08 (1H, $m, \mathrm{H}-$ 7b), 1.01 (3H, s, H-17), 0.99 (1H, $m, \mathrm{H}-7 \mathrm{a})$, 0.98 (1H, m, H-6a \& H-14a), 0.87 (3H, s, H20). ${ }^{13} \mathrm{C}-\mathrm{NMR} \delta$ (ppm): 151.2 (C-15), 108.8 (C-16), 73.4 (C-3), 62.3 (C-4), 56.5 (C-18), 54.7 (C-10), 41.8 (C-8), 38.9 (C-14), 37.4 (C9), 36.2 (C-13), 35.9 (C-5 \& C-11), 34.4 (C6), 32.2 (C-12), 30.8 (C-19), 29.1 (C-2), 27.9 (C-7), 22.8 (C-17), 16.8 (C-20), 15.7 (C-1).

\section{Tagalsin E (5)}

Yellow solid. m.p. $73-75{ }^{\circ} \mathrm{C} ;[\alpha]_{\mathscr{D}}^{2 \mathrm{~s}}$ $+7.85^{\circ}\left(c 0.07, \mathrm{CHCl}_{3}\right)$; IR (KBr) v $v_{\max } 2956$, 2923, 2860, 1692, 1636, 1607, 1457, 1377, $908 \mathrm{~cm}^{-1}$; EI-MS m/z (\%): $286[\mathrm{M}]^{+}(24), 271$ (22), 243 (22), 202 (30), 189 (25), 161 (27), 149 (66), 121 (70), 107 (100), 93 (88), 79 (85); HRFABMS $m / z$ 287.2368 $[\mathrm{M}+\mathrm{H}]^{+}$(calcd for $\left.\mathrm{C}_{20} \mathrm{H}_{31} \mathrm{O}, 287.2369\right) .{ }^{1} \mathrm{H}-\mathrm{NMR} \delta$ (ppm): 5.92 (1H, brs, H-18a), 5.79 (1H, dd, $J=17.5,10.8$ 
$\mathrm{Hz}, \mathrm{H}-15), 5.24$ (1H, brs, H-18b), 4.92 (1H, $d$, $J=17.5 \mathrm{~Hz}, \mathrm{H}-16 \mathrm{a}), 4.84(1 \mathrm{H}, d, J=10.8 \mathrm{~Hz}$, $\mathrm{H}-16 \mathrm{~b}), 2.00$ (1H, $m, \mathrm{H}-1 \mathrm{a}), 2.11$ (1H, $m, \mathrm{H}-$ 1b), $2.54(1 \mathrm{H}, d d, J=13.5,5.0), 2.53(1 \mathrm{H}, d d$, $J=13.5,8.5, \mathrm{H}-2 \mathrm{a}), 2.15$ (1H, $m, \mathrm{H}-6 \mathrm{~b}), 1.69$ $(1 \mathrm{H}, d d d, J=13.0,3.5,3.5 \mathrm{~Hz}, \mathrm{H}-11 \mathrm{~b}), 1.52$ $(1 \mathrm{H}, d d d, J=13.0,13.0,3.5 \mathrm{~Hz}, \mathrm{H}-12 \mathrm{~b}), 1.48$ $(1 \mathrm{H}, d d d, J=15.0,14.0,2.5 \mathrm{~Hz}, \mathrm{H}-16 \mathrm{a}), 1.40$ $(1 \mathrm{H}, d d d, J=11.5,2.0,2.0 \mathrm{~Hz}, \mathrm{H}-10 \mathrm{a}), 1.34$ (1H, $m, \mathrm{H}-10 \mathrm{~b}), 1.32$ (1H, $m, \mathrm{H}-7 \mathrm{~b}), 1.30(1 \mathrm{H}$, $m, \mathrm{H}-14 \mathrm{~b}), 1.22$ (1H, ddd, $J=13.0,3.5,3.0$ Hz, H-12a), 1.12 (1H, $m$, H-7a \& H-11a), 1.08 (3H, $s, \mathrm{H}-19), 1.02$ (3H, $s, \mathrm{H}-17), 0.98(1 \mathrm{H}, m$, $\mathrm{H}-14 \mathrm{a}), 0.78$ (3H, $s, \mathrm{H}-20) .{ }^{13} \mathrm{C}-\mathrm{NMR} \delta$ (ppm): 203.7 (C-3), 151.2 (C-15), 152.5 (C-4), 116.5 (C-18)109.1 (C-16), 52.6 (C-10), 42.7 (C-8), 41.2 (C-5), 39.2 (C-14), 38.4 (C-9), 37.6 (C6), 36.7 (C-2), 36.6 (C-13), 35.8 (C-11), 33.8 (C-19), 32.1 (C-12), 25.8 (C-7), 23.2 (C-17), 18.0 (C-1), 13.9 (C-20).

\section{Tagalsin F (6)}

White solid. m.p. $98-99{ }^{\circ} \mathrm{C} ;[\alpha]_{D}^{25}$ $+34.86^{\circ}\left(c 0.072 ; \mathrm{CHCl}_{3}\right)$; IR (KBr) $v_{\max } 3084$, 2967, 2922, 2857, 1622, 1455, 1384, $1188 \mathrm{~cm}^{-}$ 1; EI-MS m/z (\%): $303[\mathrm{M}+\mathrm{H}]^{+}$; HRFABMS $m / z, 303.2319[\mathrm{M}+\mathrm{H}]^{+}$(calcd for $\mathrm{C}_{20} \mathrm{H}_{31} \mathrm{O}_{2}$, 303.2318). ${ }^{1} \mathrm{H}-\mathrm{NMR} \delta$ (ppm): $7.90(1 \mathrm{H}, d, J=$ 7.8, H-18), $5.78(1 \mathrm{H}, d d, J=17.5,10.7 \mathrm{~Hz}, \mathrm{H}-$ 15), $4.88(1 \mathrm{H}, d, J=17.5 \mathrm{~Hz}, \mathrm{H}-16 \mathrm{a}), 4.84$ $(1 \mathrm{H}, d, J=10.7 \mathrm{~Hz}, \mathrm{H}-16 \mathrm{~b}), 2.46(1 \mathrm{H}, d d, J=$ 13.0, $3.0 \mathrm{~Hz}, \mathrm{H}-2 \mathrm{a}), 2.45(1 \mathrm{H}, d d, J=13.0$, $9.0 \mathrm{~Hz}, \mathrm{H}-2 \mathrm{~b}), 2.13(1 \mathrm{H}, d d d, J=14.0,4.0$, $3.0 \mathrm{~Hz}, \mathrm{H}-6 \mathrm{~b}), 2.04$ (1H, ddd, $J=16.5,9.0$, $7.0 \mathrm{~Hz}, \mathrm{H}-1 \mathrm{~b}), 1.97$ (1H, m, H-1a), $1.62(1 \mathrm{H}$, $d d d, J=13.0,4.0,3.0 \mathrm{~Hz}, \mathrm{H}-11 \mathrm{~b}), 1.53(1 \mathrm{H}$, $d d d, J=14.0,14.0,4.0 \mathrm{~Hz}, \mathrm{H}-12 \mathrm{~b}), 1.42(1 \mathrm{H}$, $d d d, J=14.0,13.0,4.0 \mathrm{~Hz}, \mathrm{H}-6 \mathrm{a}), 1.41(1 \mathrm{H}$, $d d d, J=13.5,13,0,3.5 \mathrm{~Hz}, \mathrm{H}-11 \mathrm{a}), 1.32(1 \mathrm{H}$, $d d, J=13.5,13.0 \mathrm{~Hz}, 14 \mathrm{~b}), 1.24(1 \mathrm{H}, m, \mathrm{H}-$ 10), $1.23(1 \mathrm{H}, m, \mathrm{H}-7 \mathrm{~b}), 1.20(1 \mathrm{H}, d d d, J=$ 14.0, 3.5, 3.0 Hz, H-12a), 1.15 (3H, s, H-19), $1.10(1 \mathrm{H}, m, \mathrm{H}-7 \mathrm{a}), 1.01$ (3H, $s, \mathrm{H}-17), 0.96$ $(1 \mathrm{H}, d d d, J=13.5,1.5,1.5 \mathrm{~Hz}, \mathrm{H}-14 \mathrm{a}), 0.69$ (3H, s, H-20). ${ }^{13} \mathrm{C}-\mathrm{NMR} \delta$ (ppm): 199.6 (C-3),
171.2 (C-18), 150.9 (C-15), 116.7 (C-4), 108.8 (C-16), 51.8 (C-10), 42.5 (C-8), 38.8 (C-14), 37.7 (C-9), 36.7 (C-6), 36.3 (C-5), 36.1 (C13), 35.8 (C-19), 35.2 (C-11), 31.7 (C-12), 31.5 (C-2), 25.5 (C-7), 23.0 (C-17), 16.1 (C1), 12.7 (C-20).

\section{Tagalsin G (7)}

Colorless oil; $[\alpha]_{D}^{25} \quad+55.97^{\circ}$ $\left(c 0.09 ; \mathrm{CHCl}_{3}\right)$; IR (KBr) $v_{\max } 3429,2924$, 2857, 1732, 1667, 1640, 1451, 1394, 1201, $1101 \mathrm{~cm}^{-1}$; EI-MS m/z (\%): $302[\mathrm{M}]^{+}(8), 287$ (43), 263 (53), 203 (100), 149 (73), 107 (89), 93 (55), 81 (56); HRFABMS $m / z 303.2319$ $[\mathrm{M}+\mathrm{H}]^{+}$(calcd for $\left.\mathrm{C}_{20} \mathrm{H}_{31} \mathrm{O}_{2}, 303.2318\right) .{ }^{1} \mathrm{H}-$ NMR $\delta(\mathrm{ppm}): 5.81(1 \mathrm{H}, d d, J=17.5,10.8$ $\mathrm{Hz}, \mathrm{H}-15), 4.94$ (1H, $d, J=17.5 \mathrm{~Hz}, \mathrm{H}-16 \mathrm{a})$, $4.87(1 \mathrm{H}, d, J=10.8 \mathrm{~Hz}, \mathrm{H}-16 \mathrm{~b}), 2.87(1 \mathrm{H}$, $d d, J=18.7,6.5 \mathrm{~Hz}, \mathrm{H}-1 \mathrm{~b}), 2.77(1 \mathrm{H}, d, J=$ $18.7 \mathrm{~Hz}, \mathrm{H}-1 \mathrm{a}), 2.19(1 \mathrm{H}, d d d, J=13.5,2.5$, $2.5 \mathrm{~Hz}, \mathrm{H}-6 \mathrm{~b}), 1.90$ (3H, $s, \mathrm{H}-18), 1.68(1 \mathrm{H}, d$, $J=6.5 \mathrm{~Hz}, \mathrm{H}-10), 1.66(1 \mathrm{H}, d d d, J=13.5$, $3.5,3.5, \mathrm{H}-11 \mathrm{~b}), 1.54(1 \mathrm{H}, d d d, J=13.5,13.5$, $4.0 \mathrm{~Hz}, \mathrm{H}-12 \mathrm{~b}), 1.53$ (1H, m, 7b), 1.41 (1H, $m$, $\mathrm{H}-8), 1.40(1 \mathrm{H}, d d, J=13.5,13.0 \mathrm{~Hz}, \mathrm{H}-14 \mathrm{~b})$, 1.30 (1H, $m, \mathrm{H}-6 \mathrm{a}), 1.27$ (3H, $s, \mathrm{H}-19), 1.21$ $(1 \mathrm{H}, d d d, J=13.5,4.0,3.5 \mathrm{~Hz}, \mathrm{H}-12 \mathrm{a}), 1.18$ (1H, m, H-7a), $1.12(1 \mathrm{H}, d d d, J=13.5,13.5$ $\mathrm{Hz}, 4.0 \mathrm{~Hz}, \mathrm{H}-11 \mathrm{a}), 1.05$ (3H, $s, \mathrm{H}-17), 1.02$ (1H, $m, \mathrm{H}-14 \mathrm{a}), 0.62$ (3H, $s, \mathrm{H}-20) .{ }^{13} \mathrm{C}-\mathrm{NMR}$ $\delta$ (ppm): 193.1 (C-2), 150.9 (C-15), 144.5 (C3), 135.5 (C-4), 108.9 (C-16), 54.5 (C-10), 41.6 (C-8), 39.0 (C-5), 38.9 (C-14), 38.1 (C9), 38.0 (C-6), 36.2 (C-13), 34.2 (C-11), 33.2 (C-1), 31.7 (C-12), 31.6 (C-19), 26.7 (C-7), 23.1 (C-17), 13.7 (C-20), 11.6 (C-18).

\section{Caspase-3-activity}

Caspase- 3 calorimetric assay was used to measure the protease activity of caspase 3 under the activation of seven dolaboranes namely tagalsins $\mathbf{1}, \mathbf{2}, \mathbf{3}, \mathbf{4}, \mathbf{5}, \mathbf{6}$ and $\mathbf{7}$. The procedure reported by Chacha (2011) was adopted with minor modification. 


\section{RESULTS}

The chromatographic analyses of the chloroform extracts from the roots of Ceriops tagal yielded seven dolaborane-diterpenes identified as tagalsins A (1), B (2), C (3), D (4), E (5), F (6) and G (7) (Fig 1). The chemical structures of these compounds were established on the basis of IR, MS and NMR spectral data and in comparison with the existing data in literature (Zhang et al., 2005).

Tagalsins A - G $(\mathbf{1}-\mathbf{7})$ were evaluated for the activation caspase-3 enzyme in cleaving $p$-nitroaniline from acetyl-Asp-GluVal-Asp $p$-nitroanilide (Ac-DEVD-pNA) in the presence of the caspase-3 inhibitor AcDEVD-CHO. The released $p$-NA was detected microplate reader set at $405 \mathrm{~nm}\left(\varepsilon_{\mathrm{mM}}=10.5\right)$ and the concentration of the released $p$-NA was calculated from the absorbance mean values. The minimum activation concentration (MAC) of the compounds was established. The cleavage of the caspase-3-specific colorimetric substrate (DEVD-pNA) was therefore considered to represent caspase-3 activity.

Tagalsins A - G activated caspase-3 activity in cleaving $p$-NA from Ac-DEVD$p$ NA which is an important biochemical step for the execution of apoptosis. Tagalsin A (1) was the most active followed by tagalsin B (2) and tagalsin E (5) was the least active (Table 1). The minimum activation concentration (MAC) of tagalsins A - G (1 - 7) were 0.12 , $0.15,0.43,0.25,1.16,0.54$ and $0.62 \mathrm{mg} / \mathrm{ml}$ which gave 81.4, 72.5, 32.8, 68.4, 13.7, 27.1 and $24.6 \mu \mathrm{mol} / \mathrm{ml}$ of cleaved $P$-NA respectively. These compounds were tested for in vitro cytotoxicity against cultured human tumor cell lines H-60, Bel-7402 and Hela, they did not show any activity (Zhang et al, 2005). The anticancer activity exhibited in this study indicates that tagalsins $A-G$ are specific in action and thus offers an avenue for the development of novel anticancer agents. This is the first time for tagalsins $A-G$ to be evaluated for the induction of apoptosis through activation of caspase-3 enzyme.

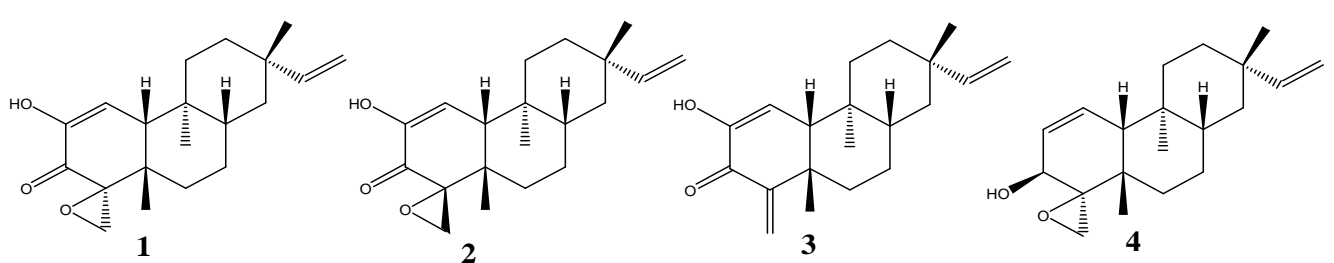

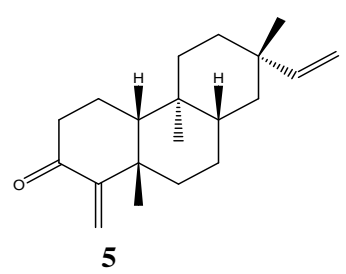<smiles>C=CC1(C)CC[C@H]2[C@@](C)(CCC3(C)C(C(C)=C(C)C)C(=O)C=C[C@@]23C)C1</smiles>

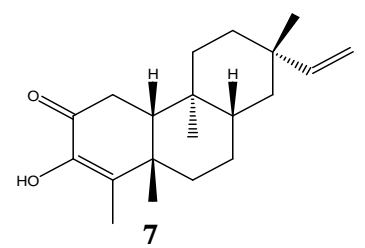

Figure 1: Structures of tagalsin A (1), B (2), C (3), D (4), E (5), F (6) and G (7). 
Table 1: Minimum activation concentration (MAC) of compounds 1, 2, 3, 4, 5, 6 and 7 with the concentrations of the released $P$-NA.

\begin{tabular}{|c|c|c|c|c|c|c|c|}
\hline Compounds & 1 & 2 & 3 & 4 & 5 & 6 & 7 \\
\hline $\mathrm{MAC}(\mathrm{mg} / \mathrm{ml})$ & 0.12 & 0.15 & 0.43 & 0.25 & 1.16 & 0.54 & 0.62 \\
\hline$[P-\mathrm{NA}] \mathrm{mol} / \mathrm{ml})$ & 81.4 & 72.5 & 32.8 & 68.4 & 13.7 & 27.1 & 24.6 \\
\hline
\end{tabular}

\section{DISCUSSION}

The available anticancer agents are mostly targeting processes that cancer cells use to grow and divide, such as the ability of the cells to replicate their deoxyribose nucleic acid (Malpas, 1997; Chen et al., 2000; Braybroke et al., 2003; Usui et al., 2004; Dias et al., 2005; Kamal et al., 2005). With the establishment that cancerous cells produces proteins that blocks the process of apoptosis, the development of compounds that activates the apoptosis of cancerous cells has emerged as a powerful strategy in the fight against cancer. Thus, tagalsins A - G (1 - 7) isolated from the roots of Ceriops tagal were tested for the induction of caspase- 3 enzyme in the activation of apoptotic pathway. The difference in the activity exhibited by these compounds is probably due to the difference in their structures (Figure 1 and Table 1).

Structure activity relationship has revealed that epoxide rings and oxygen atoms are important in the activation of caspase-3. This was proved by the high activity exhibited by tagalsins A (1) and B (2) which posses epoxide rings and two oxygen atoms each. Tagalsin D (4) which has an epoxide ring and one hydroxyl group displayed higher activity than tagalsins $\mathrm{C}(\mathbf{3}), \mathrm{E}(\mathbf{5}), \mathrm{F}(\mathbf{6})$ and $\mathrm{G}(\mathbf{7})$. Tagalsin E (5) which has only one oxygen atom was the least active proving the importance of oxygen atoms in the activity of dolaboranes. The present study corroborates a previous report that oxygenation enhance the activity of isopimaranes in inducing caspase- 3 enzyme in the activation of apoptotic pathways (Chacha, 2011). This is the first time for tagalsins $1-7$ to be evaluated for the induction of apoptosis through the activation of caspase- 3 enzyme.

\section{Conclusion}

Chemotherapeutic agents based on natural products have been the backbone of healthcare systems for all communities since ancient times, and indeed the treatment of cancer has relied heavily on therapeutics based on natural products and their analogues. This study has established that dolaboranes from the roots of Ceriops tagal induces caspase-3 activity in activating apoptosis in cancerous cells and thus providing unique mechanism of actions.

\section{ACKNOWLEDGEMENTS}

This study was supported by the Western Indian Ocean Regional Initiative in Marine Science and Education (WIORISE) through faculty research fund. Mwadini Mtumwa is acknowledged for identification of plant materials. The author appreciates Kisakasaka Mangrove Conservation Group for the permission to collect plant materials in the Kisakasaka Mangrove Reserve Forest.

\section{REFERENCES}

Braybrooke JP, Levitt NC, Joel S, Davis T, Madhusudan S, Turley H, Wilner S, Harris AL, Talbot DC. 2003. Pharmacokinetic study of cisplatin and infusional etoposide phosphate in advanced breast cancer with correlation of response to topoisomerase II a-expression. Clin. Cancer Res., 9(13): 4682-4688. 
Chacha M. 2011. Terpenoids from the roots of Ceriops tagal induces apoptosis through activation of caspase-3 enzyme. Int. J. Biol. Chem. Sci., 5(2): 402-409.

Chen YM, Perny RP, Yang KY, Liu TW, Tsai CM, Ming-Liu J, Whang-Peng J. 2000. A multicenter phase II trial of vinorelbine plus gemcitabine in previously untreated inoperable (stage IIIB/IV) non small cell lung cancer. Chest., 117(6): 1583-1589.

Dias LC, de Oliveira LG, de Sousa MA, Ellensohn RH. 2005. Total synthesis of the potent immunosuppressant (-)-pironetin. Arkivoc, vi: 62-87.

Hanahan, Weinberg RA. 2000. The hallmarks of cancer. Cell, 100: 57-70.

Hu WM, Li MY, Li J, Xiao Q, Feng G, Wu J. 2010. Dolabranes from the Chinese mangrove, Ceriops Tagal. J. Nat. Prod., 73(10): 1701-1705.

Igney FH, Krammer PH. 2002. Death and antideath: tumor resistance to apoptosis. Nat. Rev. Cancer., 2(4): 277-288.

Kamal A, Gayatri NL, Reddy DR, Reddy PSM, Arifuddin M, Dastidar SG, Kondapi AK, Rajkumar M. 2005. Synthesis of biological evaluation of new 4b-imido-substituted podophyllotoxin congeners. Bioorg. Med. Chem., 13(22): 3338-3343.

Kirkin V, Joos S, Zornig M. 2004. The role of Bcl-2 family members in tumorigenesis.
Biochim. Biophys. Acta., 1644(2-3): 229249.

Lowe SW, Cepero E, Evan G. 2004. Intrinsic tumor suppression. Nature, 432: 307-315.

Peterson QP, Goode DR, West DC, Ramsey1 KN, Lee1 JJL, Paul J, Hergenrother PJ. 2009. PAC-1 activates procaspase-3 in vitro through relief of zinc-mediated inhibition. Journal of Molecular Biology, 388(1): 144-158.

Soengas MS, Capodieci P, Polsky D, Mora J, Esteller M, Opitz-Araya X. McCombie R, Herman JG, Gerald WL, Lazebnik YA, Cordón-Cardó C, lowe SW. 2001. Inactivation of the apoptosis effector Apaf1 in malignant melanoma. Nature, 409(6817): 207-211.

Usui T, Watanabe H, Nakagama H, Kanoh N, Kondoh M, Asao T, Takio K, Nishikawa K, Kitahara T, Osaka H. 2004. The anticancer natural product pironetin selectively targets LY352 of the alpha tubulin. Chem. Biol., 11(6): 799-806.

Vogelstein B, Kinzler KW. 2001. Achilles' heel of cancer. Nature, 412(6850): 865-866.

Zhang Y, Deng ZW, Gao TX, Proksch P, Lin WH. 2005. Tagalsins A-H, dolabrane-type diterpenes from the mangrove plant, Ceriops tagal. Phytochemistry, 66(12): 1465-1471. 